Original Article

\title{
Epidemiology of Extrapulmonary Tuberculosis in the Golestan Province, Northeast of Iran
}

\author{
Alireza Nikonajad \\ Department of infectious disease, \\ Faculty of Medicine, Qazvin University \\ of Medical Sciences, Qazvin, Iran \\ Sadegh Ali Azimi \\ Department of infectious disease, \\ Faculty of Medicine, Qazvin University \\ of Medical Sciences, Qazvin, Iran \\ Abbas Allami \\ Department of infectious disease, \\ Faculty of Medicine, Qazvin University \\ of Medical Sciences, Qazvin, Iran \\ Reza Qasemi Bargi \\ Department of infectious disease, \\ Faculty of Medicine, Qazvin University \\ of Medical Sciences, Qazvin, Iran \\ Alijan Tabarraei \\ Infectious diseases research Center, \\ Golestan University of Medical \\ Sciences, Gorgan, Iran \\ Corresponding author: Abbas Allami \\ Address: Department of infectious \\ disease, Faculty of Medicine, Qazvin \\ University of Medical Sciences, \\ Qazvin, Iran \\ Email: allami@qums.ac.ir \\ Received: $2019 / 07 / 4$ \\ Revised: 2019/08/7 \\ Accepted: $2019 / 08 / 7$ \\ (c) (i) (8) \\ This work is licensed under a Creative \\ Commons Attribution 4.0 License.
}

DOI: $10.29252 / \mathrm{mlj} .15 .1 .1$

\begin{abstract}
Background and objectives: Although extrapulmonary tuberculosis (EPTB) is a secondary target for national TB control programs, it is considered an important health problem worldwide. This study was carried out to investigate the epidemiology of EPTB in the Golestan Province, Northeast of Iran.
\end{abstract}

Methods: This was a population-based, retrospective analysis of all EPTB cases registered in the TB Unit of the Golestan Province during 2012-2015. Socioeconomic and environmental variables as well as data regarding place of residence, method of admission and diagnosis and outcome status were recorded. Data were analyzed in SPSS 25.0 using descriptive and analytical statistics.

Results: A total of 741 EPTB cases were included in the study. Patients were mainly female (59.1\%), aged under 40 years $(57.6 \%)$, Fars $(39.6 \%)$ and with less than five years of education $(46.7 \%)$. The prevalence of EPTB was significantly higher in non-native ethnicities $(\mathrm{P}<0.001)$. The mortality rate among patients was $5.1 \%$. In addition, the prevalence of EPTB was significantly higher among smokers $(P<0.001)$. The most common forms of the disease were pleural $(30.5 \%)$ and lymphadenopathy (22.0\%). Culture and PCR were performed in only about 10 percent of the EPTB patients.

Conclusion: EPTB is more prevalent among the non-native population of the Golestan Province. Promoting socioeconomic status and development of screening programs may help reduce the prevalence of EPTB in this area.

Keywords: Tuberculosis, Epidemiology, Immigration, Iran 


\section{INTRODUCTION}

Tuberculosis (TB) is one of the oldest human diseases that still remains a leading cause of death worldwide (1). In 2017, ten million people developed TB which resulted in an estimated 1.6 million deaths. The disease burden caused by TB is falling in most countries but not fast enough to reach the first milestones of the World Health Organization (WHO) End TB Strategy (2). Extrapulmonary TB (EPTB) can occur in up to one-third of cases and affects organs other than the lungs (3). It is an important health problem due to the high mortality and morbidity rates. In 2017, $14 \%$ of the 6.4 million TB cases developed EPTB. The prevalence of EPTB ranges from $8 \%$ in the Western Pacific region to $24 \%$ in the Eastern Mediterranean region (2). From 2012 to 2016, 20-29\% of all Iranian TB cases reported to WHO had been diagnosed with EPTB. It should be noted that patients with EPTB are more likely to be underreported (4). The epidemiology of EPTB in the northeastern part of Iran is not clear (59 ). One study was focused on seasonal pattern of the disease in this region and another reported only 15 cases of EPTB between 2008 and $2013(10,11)$. The present study was carried out to determine the epidemiology of EPTB in the Golestan Province, Northeast of Iran.

\section{MATERIALS AND METHODS}

This was a population-based, retrospective analysis of all cases of EPTB reported to the TB Unit of the Golestan Province from January 2012 to December 2015. The Golestan Province borders Turkmenistan and is one of the semi-developed areas of Iran (12). Sociodemographic variables including gender, ethnicity and educational level as well as environmental variables including place of residence and history of imprisonment were recorded. Comorbidities or history of exposure to environmental risk factors such as HIV/AIDS, smoking, diabetes and other diseases were also evaluated. Clinical features including extrapulmonary sites, method of diagnosis (histopathology, culture, imaging and PCR), admission type (new case, relapse, readmission or transfer), anti-TB treatment administered under Directly Observed Therapy (DOT) and outcome were also included in the analysis. Clinical forms of TB were classified according to the WHO definitions.
Accordingly, EPTB was defined as TB affecting organs and tissues outside the lungs. Patients with both pulmonary and EPTB were classified as a case of pulmonary TB (13).

Sampling was done through census. Lack of data in the variable "clinical forms of TB" and ambiguity in the diagnosis was the exclusion criteria. Primary data were extracted from the TB registered data of Golestan Province. To complete the questionnaire information (missing data and treatment outcomes of patients), we followed up patients for at least one year after completion of therapy. Data were collected using a checklist and then analyzed using SPSS statistical software package (version 25.0, SPSS Inc., Chicago IL, USA). The distribution of gender, ethnicity and smoking among EPTB patients was compared with that of among the general population of the province using the $\chi^{2}$ test. A p-value of less than 0.05 was considered as statistically significant.

The study was approved by the Research Ethics Committee of Qazvin University of Medical Sciences, Iran (approval code: IR.QUMS.REC.1396.30). The institutional review granted permission for use of information provided by the national TB data.

\section{RESULTS}

Overall, 782 TB cases were identified during the study period. Of these cases, 37 $(4.75 \%)$ were included in the study based on EPTB diagnosis. Table 1 shows different features and characteristics of the EPTB cases in both male and female patients. The continuous variables were not normally distributed in our dataset. Thus, we reported these variables as the median and interquartile range (IQR).

Of EPTB patients, $59.1 \%$ were female, $57.6 \%$ were under 40 years old and $46.7 \%$ had less than five years of education. All patients were treated under DOT and $93.8 \%$ of the cases completed the course of treatment. Smoking was significantly more frequent among EPTB patients compared to the general population ( $13 \%$ vs. $5.5 \%, \mathrm{P}<0.001)$. Only one of the patients was co-infected with HIV, but the HIV status of most EPTB patients $(89.3 \%)$ was unknown. Moreover, EPTB was significantly more prevalent in females than in males $(\mathrm{P}<0.0001$, Table 1$)$. The most common forms of EPTB were pleural (30.5\%), lymphadenopathy (22.0\%), bone and joint TB 
(9.3\%). The most common sites of involvement were breast (69 cases, 9.3\%), peritoneum and gastrointestinal (55 cases, 7.4\%), genitourinary (43 cases, 5.8\%), meningeal/CNS (24 cases, 3.2\%), (15 cases, $2.6 \%$ ), cutaneous (15 cases, $2.0 \%$ ), renal (5 cases, $0.9 \%$ ), ophthalmic (12 cases, 1.6\%) and other sites (40 cases, $5.4 \%$ ).

The prevalence of EPTB was more prevalent in the non-native population than in the native population (Table 2). Distribution of ethnicities in the Golestan Province is as follows: Fars (40\%), Turkmen (33.1\%), Sistani (15.4\%), Baluchi $(4.3 \%)$ and other ethnicities including Kurd and Kazakhs (7\%). The frequency of EPTB patients in the province based on ethnicity was as follows: Fars (34\%), Turkman (13.9\%), Sistani (28.9\%), Baluchi
$(8.5 \%)$ and others $(1.9 \%)$.

A significant difference was also observed in the age distribution of EPTB cases $(\mathrm{P}<0.001)$. The mean age of bone and lymph TB patients was 48 and 34 years, respectively. Table 3 shows the method of diagnosis and treatment outcome of EPTB patients.

The maximum and minimum delay observed were related to genitourinary (4.8 months) and skin TB (1.9 months), respectively. The most and least common diagnosis methods were histopathological examination (393 cases, 53.0\%) and PCR (35 cases, 4.7\%), respectively (Figure 1). Mycobacterial culture and PCR were performed in about 10 percent of EPTB patients.

Table 1. Sociodemographic characteristics of EPTB patients in the Golestan Province (Iran) during 2012-2015

Sex

Median (IQR) or n (\%)

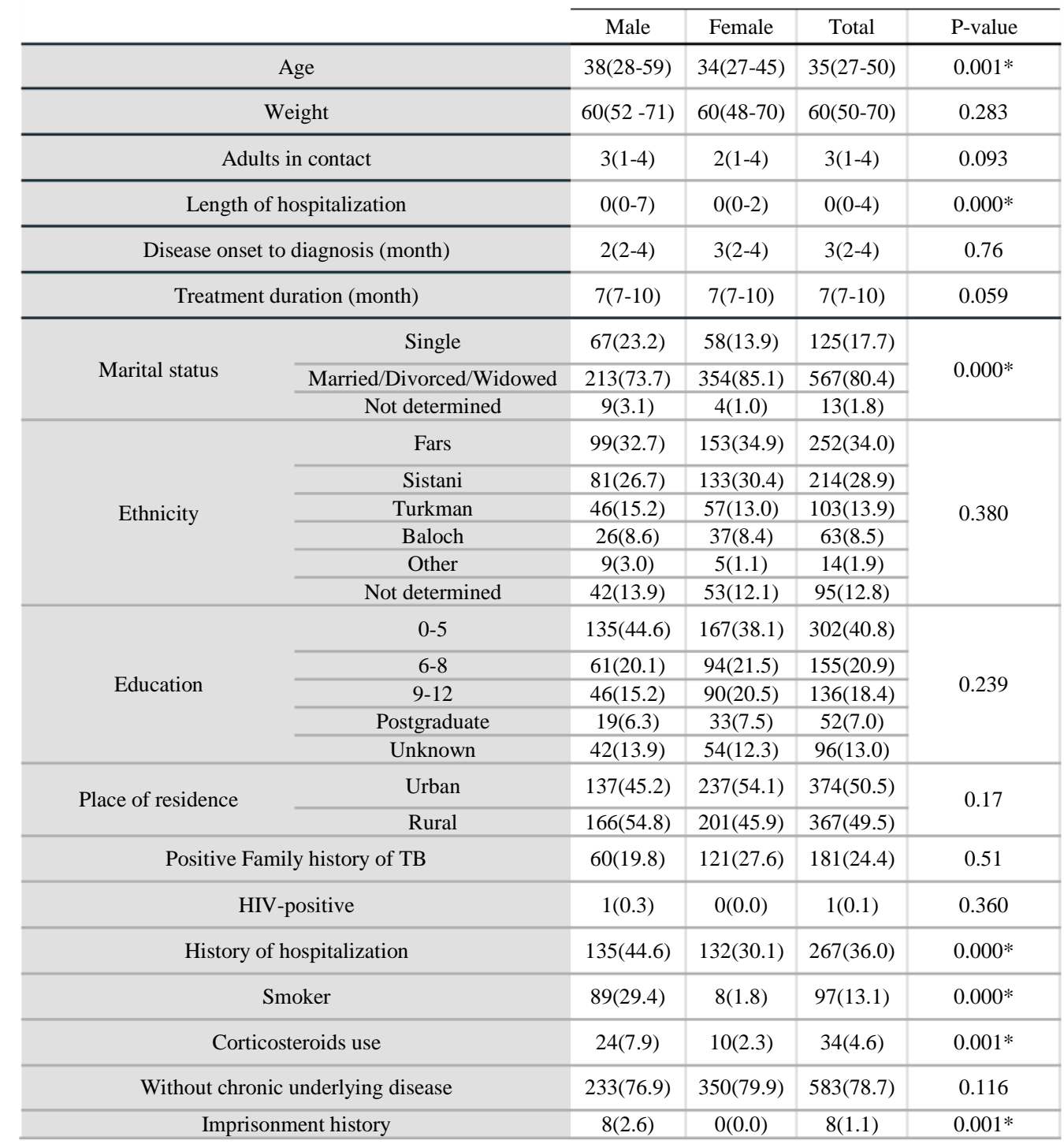

*: statistically significant based on the Mann-Whitney U or Chi-Square tests. NOTE. Data are median (IQR) or number (\%) of patients, unless otherwise indicated. 
Table 2. Distribution of EPTB cases in the Golestan Province based on ethnicity and estimating the goodness of fit of ethnicity distributions

\begin{tabular}{|c|c|c|c|c|}
\hline & Observed $\mathrm{N}$ & Expected N & Residual & Test \\
\hline Sistani & 214 & 99.7 & 114.3 & \multirow{5}{*}{$\begin{array}{l}\text { Chi-Square }=255.120 \\
D f=4 \\
\text { P-value }=0.000^{*}\end{array}$} \\
\hline Baloch & 63 & 27.8 & 35.2 & \\
\hline Fars & 252 & 258.9 & -6.9 & \\
\hline Turkman & 103 & 214.3 & -111.3 & \\
\hline Other & 14 & 45.3 & -31.3 & \\
\hline Total & 646 & & & \\
\hline
\end{tabular}

Table 3. Clinical features and treatment outcomes of EPTB cases in the Golestan Province during 2012-2015

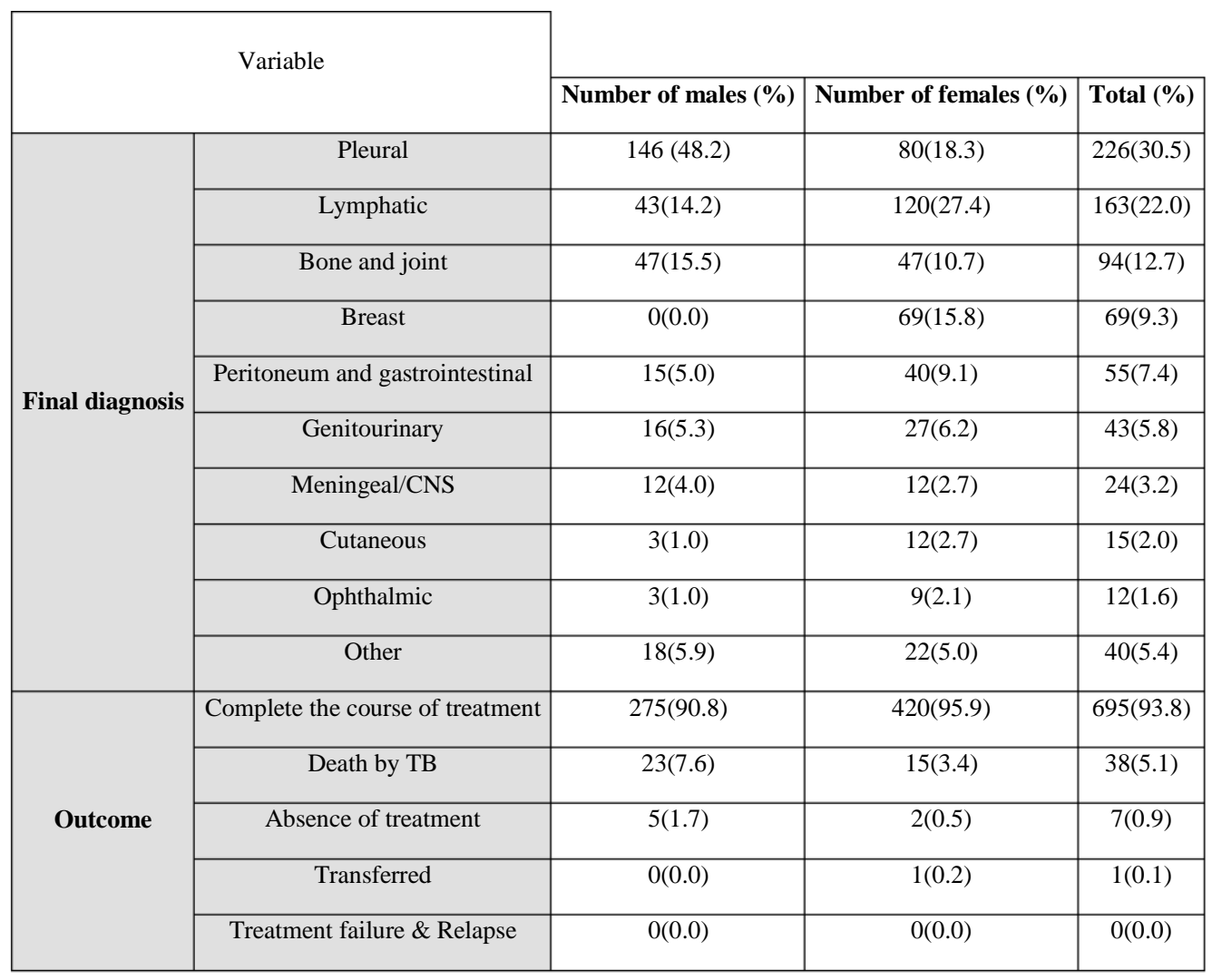

Figure 1. The frequency distribution of different methods of EPTB diagnosis in the Golestan Province during 2012-2015.

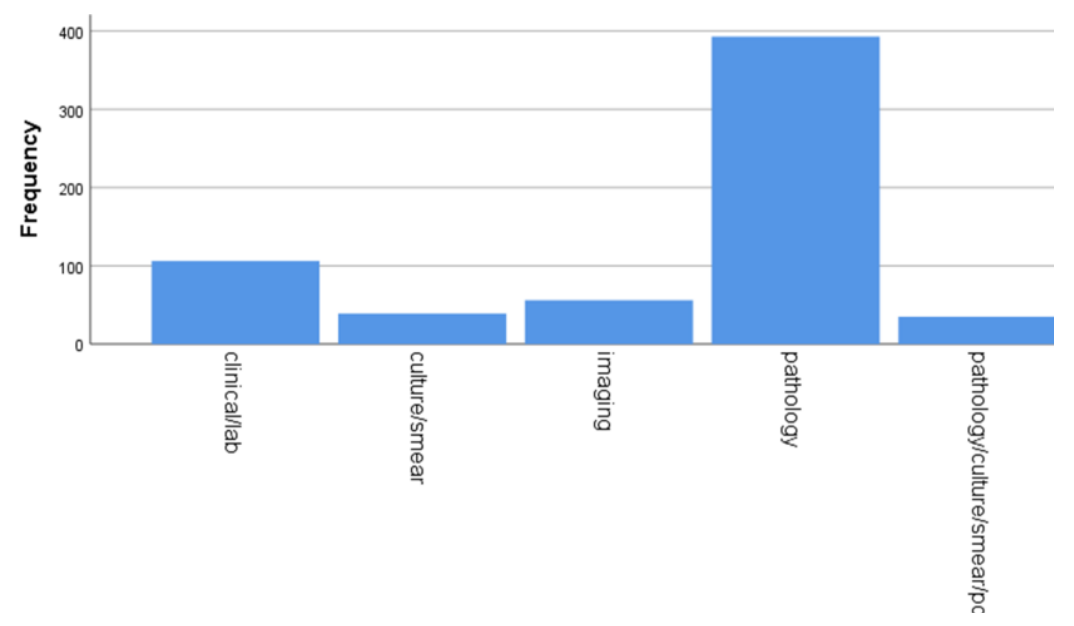




\section{DISCUSSION}

The present study evaluated the epidemiology of EPTB in the Golestan Province, Northeast of Iran. The province ranks second in the country in terms of EPTB incidence. In 2017, the incidence of EPTB was 2.91 per 100,000 population countrywide and 8.58 per 100,000 people in the Golestan Province (4). It seems that TB is related to socioeconomic status $(3,13)$. During the study period, the incidence of $\mathrm{TB}$ in the Golestan Province and Iran decreased from 51.47 and 14.43 per 100000 in 2012 to 38.26 and 12.59 per 100000 in 2017, respectively (14). However, the EPTB incidence rate in most provinces of the country has not decreased in the past ten years $(4,14)$. It is well known that EPTB comprises a significant proportion of TB patients (2). However, since EPTB is less infectious compared to other forms of $\mathrm{TB}$, it may not receive the attention required for the development of national strategies and control programs, particularly in developing countries (15).

One epidemiological study on EPTB in Brazil demonstrated that a higher education level was associated with a higher frequency of EPTB. The authors attributed this result to easier access to diagnostic and health services among more educated individuals given that the diagnosis of EPTB often requires invasive sampling procedures with additional expense and risk, and the collection of such samples is more difficult in public health services of resource-constrained regions (15). However, our results demonstrated that the frequency of EPTB was higher among individuals with a low education level. This result may be related to a the high rate of illiteracy in the Golestan Province (17.4\% in men and $33.8 \%$ in women) (14).

More than half of the patients were under 40 years of age at the time of diagnosis, which is similar to previous reports $(16,17)$. In our study, $49.5 \%$ of cases were in rural areas and $50.5 \%$ were in urban areas. Similar to previous studies, the incidence of EPTB was higher in women $(3,16)$.

Immigration from a high prevalence area (e.g. Zabol) is a major predisposing factor for the high prevalence of TB in this province $(5,18)$. The prevalence of EPTB was significantly higher among the Sistani and Baluchi ethnic groups. Considering the ethnic diversity of this province, Sistani and Baluchi patients account for more than $37.4 \%$ of all EPTB cases in this area, while they constituted only $19.6 \%$ of the total population of the province. Eastern parts of the province are at greater risk for TB due to their ethnic composition (Sistan and Baluchestan immigrants). It is maybe due to the higher socioeconomic status and better care with regular health service received by the native population (19). However, DOT's population coverage has increased in Iran to near $100 \%$ since 2002. According to the national program, children should be vaccinated for BCG at birth and patients with persistent cough should be examined for pulmonary TB (20). Other studies also found that EPTB is more prevalent among particular ethnic groups $(21,22)$.

In line with most studies, pleural and lymphadenopathy were the most common forms of $\operatorname{EPTB}(15,19,21)$. The most frequent site of EPTB was the pleura, followed by the peripheral lymph nodes. Pleural TB was also found as the most prevalent form of EPTB in Poland (36\%) and Romania (58\%) (23), whereas lymph nodes were reported as the most common site of EPTB in the United Kingdom (37\%) and the United States (40\%) $(21,24)$. In a recent study in China, osteoarticular involvement was the most frequent site of EPTB. These inconsistencies could be due to the difference in age of patients. Lymphatic TB is more frequently observed in children under 15 years of age (16). Moreover, it seems that pleural TB occurs as an early manifestation of primary Mycobacterium tuberculosis infection and may serve as a sentinel event in recent transmission studies (25).

Unfortunately, $89.3 \%$ of the cases of EPTB were not tested for HIV. It is recommended to develop strategies for testing these patients for HIV and to ensure that the test results are systematically included in the information databases as soon as they become available. It is known that HIV/AIDS infection is a key risk factor of EPTB. Central nervous system TB is eightfold more common in HIV-infected patients, while genitourinary TB, osteoarticular TB and lymphadenopathy were seen more often in immunocompetent patients. Other forms of EPTB are not influenced by HIV infection (26). National TB control program must pay more attention to the coincidence of TB and HIV, educate the society 
about this issue and provide necessary services to patients with HIV/AIDS.

Unfortunately, in our province, the median (IQR) of interval from onset to diagnosis was three (2-4) months. Delayed diagnosis results in increased risk of morbidity and mortality. Early diagnosis and immediate initiation of treatment are essential for the improvement of EPTB outcome. Repeated visits at the same healthcare level, resulting in nonspecific antibiotic therapy and failure to provide specialized TB services are core problems of delayed diagnosis or treatment (27).

Accurate diagnosis of EPTB requires high clinical suspicion, special diagnostic procedures, special staining procedures and culture media for acid-fast bacilli. Healthcare workers must be aware of the various forms of EPTB, particularly in endemic areas (19). Mycobacterial cultures and PCR are not universally performed during the evaluation of EPTB suspects and for this reason, these two methods were performed in a minor proportion of EPTB patients in our region. The only rapid test currently recommended by the WHO for the diagnosis of TB is the Xpert ${ }^{\circledR} \mathrm{MTB} / \mathrm{RIF}$ assay. It can provide results within two hours and was initially recommended in 2010 for the diagnosis of pulmonary TB in adults. Since 2013, it has been also recommended for diagnosing specific forms of EPTB (2).

In our study, the overall mortality rate among EPTB patients was $5.1 \%$. In a study in the US, the overall mortality rate was reported to be $15 \%$ among patients with EPTB (17).

\section{CONCLUSION}

Based on the results, EPTB is more prevalent among the non-native population of the Golestan Province, Northeast of Iran. For better identification of EPTB cases, special diagnostic methods like culture and PCR must be carried out but unfortunately, the resources to perform such tests are limited in the province. Improved diagnostic capacity is essential for better case finding and management of EPTB. Finally, promoting socioeconomic status and development of screening programs may help reduce the prevalence of EPTB in this area.

\section{ACKNOWLEDGMENTS}

We would like to thank the Health section of Golestan University of Medical Science for providing the data.

\section{CONFLICT OF INTEREST}

The authors declare that there is no conflict of interest regarding publication of this article.

\section{REFERENCES}

1.Rao B. A Study on Factors Influencing Magnitude of HIV among Patients with Diagnosed Tuberculosis. Indian Journal of Public Health Research Development. 2017;8(3). [Google $\underline{\text { Scholar] }}$

2.Global Tuberculosis Report 2018. Geneva: World Health Organization; Licence: $\quad C C \quad B Y-N C-S A \quad 3.0 \quad I G O$. https://www.who.int/tb/publications/global_report/en/ Date accessed: March 22, 2019. [Google Scholar]

3.Ramirez-Lapausa M, Menendez-Saldana A, NogueradoAsensio A. Extrapulmonary tuberculosis: an overview. Rev Esp Sanid Penit. 2015;17:3-11. [Google Scholar]

4. Ministry of Health of Iran. National Center for Tuberculosis and Leprosy Control web site; 2017 [cited 2019]; Available from: http://tblep.behdasht.gov.ir/TB_HIV_Situation_in_Iran.aspx. [ [ $\underline{\text { Google }}$ Scholar]

5.Salek S, Salek S, Emami H, Masjedi MR, Velayati AA. Epidemiologic status of tuberculosis in Golestan province. Tanaffos. 2008;7(3):63-8. [PubMed] [Google Scholar]

6.Yazdani-Charati J, Sheykholeslami AS, Kamalinia H, Sheikhi M, Rahimi E. Epidemiologic Study of Pulmonary Tuberculosis in Golestan Province, Iran 2007-2016. Journal of Mazandaran University of Medical Sciences. 2018;28(165):118-28. [Google Scholar]

7.Rafiee S, Besharat S, Jabbari A, Golalipour F, Nasermoaadeli A. Epidemiology of tuberculosis in northeast of Iran: a population-based study. Iranian Journal of Medical Sciences. 2009; 34(3): 193-7. [Google Scholar]

8.Golsha R, Mehravar F, Esboie AA, Rafiee S, Rafiee S. The Epidemiology of Skeletal Tuberculosis in Northeast of Iran: A Review of 229 Cases. Iranian journal of medical sciences. 2018; 43(4): 380. [PubMed] [Google Scholar]

9.Khodabakhshi B, Mehravar F. Breast tuberculosis in northeast Iran: review of 22 cases. BMC women's health. 2014;14(1):72. [PubMed] [Google Scholar]

10.Tahari MMH, Babaei MH, Morovati SA. Investigation and ranking of Iranian provinces in terms of access to health sector indicators. Health Information Management. 2012; 9(3): 35669. [Google Scholar]

11.Singh P, Kant S, Gaur P, Tripathi A, Pandey S. Extra Pulmonary Tuberculosis: An Overview and Review of Literature. Int J Life Sci Scienti Res. 2018; 4(1): 1539-41. [PubMed] [Google Scholar]

12.Extrapulmonary Tuberculosis in Iran according to university, 2006-2017 http://tb-lep.behdasht.gov.ir/Download\%20Files/TBEP-from-85-to-96.pdf [cited: 3/22/2019]. [Google Scholar]

13.The statics of tuberculosis (all forms) in Iran according to university, 2006-2017. http://tblep.behdasht.gov.ir/Download\%20Files/TB-total-from-85-to96.pdf [cited: 3/22/2019]. [Google Scholar]

14.Gomes T, Reis-Santos B, Bertolde A, Johnson JL, Riley LW, Maciel EL. Epidemiology of extrapulmonary tuberculosis in Brazil: a hierarchical model. BMC infectious diseases. 2014; 14(1): 9. [PubMed] [Google Scholar]

15.Table 4-population of 6 years old and more, based on sex, age, literacy and education level of Golestan province (total, urban and rural), 2017 http://amar.golestanmporg.ir/ftp/amar/sarshomari/maskan_95/ta hsilat/table04_savad_golestan.xls [Cited: 3/22/2019]. [Google Scholar]

16.Pang Y, An J, Shu W, Huo F, Chu N, Gao M, et al. Epidemiology of Extrapulmonary Tuberculosis among Inpatients, China, 2008-2017. Emerging infectious diseases. 2019;25(3):457. [PubMed] [Google Scholar] 
17. Kourbatova EV, Leonard MK, Romero J, Kraft C, del Rio C, Blumberg HM. Risk factors for mortality among patients with extrapulmonary tuberculosis at an academic inner-city hospital in the US. European Journal of Epidemiology. 2006;21(9):71521. [PubMed] [Google Scholar]

18.Metanat M, SHARIFI MB, ALAVI NR, Aminianfar M. The epidemiology of tuberculosis in recent years: Reviewing the status in south-eastern Iran. Zahedan Journal Of Research In Medical Sciences (Tabib-E-Shargh). 2012; 13(9): 1-7. [Google $\underline{\text { Scholar] }}$

19.Khazaei HA, Rezaei N, Bagheri GR, Dankoub MA, Shahryari K. Tahai A, et al. Epidemiology of tuberculosis in the Southeastern Iran. European journal of epidemiology. 2005; 20(10): 879-83. [PubMed] [Google Scholar]

20.Nasehi M, Mirhaghani L. Country Guide on TB Control. Iranian Ministry of Health, 2st ed]. Tehran, Islamic Republic of Iran, Tehran: Andishamand Publication. 2009 [Persian] http://tblep.behdasht.gov.ir/Download\%20Files/TB\%20Guidelin e\%2088.pdf.

21.Peto HM, Pratt RH, Harrington TA, LoBue PA, Armstrong LR. Epidemiology of extrapulmonary tuberculosis in the United States, 1993-2006. Clinical Infectious Diseases. 2009;49(9):1350-7. [PubMed] [Google Scholar]
22.Forssbohm M, Zwahlen M, Loddenkemper R, Rieder HL. Demographic characteristics of patients with extrapulmonary tuberculosis in Germany. European respiratory journal. 2008;31(1):99-105. [PubMed] [Google Scholar]

23.Solovic I, Jonsson J, Korzeniewska-Kosela M, Chiotan D, Pace-Asciak A, Slump E, et al. Challenges in diagnosing extrapulmonary tuberculosis in the European :.:.union:.:., 2011. Euro Surveill. 2013;18(12):pii=20432. Available online: http://www.eurosurveillance.org/ViewArticle.aspx?ArticleId=. [PubMed] [Google Scholar]

24.Kruijshaar ME, Abubakar I. Increase in extrapulmonary tuberculosis in England and Wales 1999-2006. Thorax. 2009;64(12):1090-5. [PubMed] [Google Scholar]

25.Ong A, Creasman J, Hopewell PC, Gonzalez LC, Wong M, Jasmer RM, et al. A molecular epidemiological assessment of extrapulmonary tuberculosis in San Francisco. Clinical infectious diseases. 2004;38(1):25-31. [PubMed] [Google Scholar]

26.Johansen T, Kulchavenya E, Naber K. Influence of HIV Infection on Spectrum of Extrapulmonary Tuberculosis. J Infect Dis Ther. 2018;6(377):2332-0877.1000377. [Google Scholar]

27.Storla DG, Yimer S, Bjune GA. A systematic review of delay in the diagnosis and treatment of tuberculosis. BMC Public Health. 2008;8(1):15. [PubMed] [Google Scholar]

\section{How to Cite:}

Nikonajad A, Azimi S A, Allami A, Qasemi Bargi R, Tabarraei A. [Epidemiology of Extrapulmonary Tuberculosis in the Golestan Province, Northeast of Iran]. mljgoums. 2021; 15(1): 1-7. DOI: 10.29252/mlj.15.1.1 